\title{
El potencial educativo de la lectura en la construcción de la subjetividad ${ }^{1}$
}

\section{The educational potential of reading for building subjectivity}

\author{
Juan Sáez Carreras*2 \\ juansaez@um.es \\ Margarita Campillo Díaz* \\ marga@um.es \\ José García Molina** \\ jose.gmolina@uclm.es \\ * Universidad de Murcia, España \\ * Universidad de Castilla-La Mancha, España
}

\section{Resumen:}

El punto de partida de esta exploración de carácter teórico y empírico tiene que ver con el potencial educativo que encierra la lectura, en general, y la literatura, en particular. La hipótesis que trata de confirmar es si la lectura contribuye a educar la subjetividad. El artículo tiene por objetivo dar razón de esta investigación orientada por la articulación lectura-educación-subjetividad, primera exploración realizada en la geografía castellana. Se revisan, no obstante, aquellos trabajos que han explorado en nuestro país la relación de los dos conceptos del triángulo, lectura y educa-

\begin{abstract}
:
The starting point for this theoretical and empirical piece of research comes from the idea that reading in general, and literature in particular, are underlaid by a great educational potential. The hypothesis that this essay seeks to explore is whether reading contributes to training and enriching subjectivity. This essay offers the main results obtained from the research, which was articulated around the triad reading-education-subjectivity, and which is the first of this kind undertaken in Spain. Nonetheless, those Spanish studies exploring the relationship
\end{abstract}

1 Este artículo es,en buena medida, fruto de la exploración sobre "educación, literatura y subjetividad" y fue subvencionada en el marco de colaboración entre el Centro de Formación y Desarrollo Profesional de la Universidad de Murcia y la Comunidad Autónoma de la Región de Murcia y, más concretamente, es fruto de los diversos acuerdos existentes de las dos agencias educativas, uno de cuyos productos es el Programa I, cuya filosofía se orienta, fundamentalmente, a la investigación y la innovación pedagógica. Este trabajo ha sido realizado con la colaboración del Equipo de Investigación "Intervención Socioeducativa" de la Facultad de Educación de la Universidad de Murcia y fue subvencionado con la cantidad de 3.000 euros.

2 Dirección para correspondencia (Correspondence address):

Juan Sáez Carreras. Dpto. de Teoría e Historia de la Educación. Facultad de Educación. Universidad de Murcia. Campus de Espinardo, 30100, Murcia (España). 
ción, con la finalidad de contrastar y valorar lo que el tercer elemento del mismo, la noción de subjetividad, añade a los dos anteriores abriendo una línea de investigación tan potente como innovadora para el campo de la enseñanza formal así como para la educación voluntaria y descondicionada. Para la obtención de información se ha diseñado y aplicado un cuestionario a estudiantes de titulaciones diversas así como a personas adultas a fin de contrastar percepciones de sujetos con diferente experiencia, formación y edad. La discusión de los resultados y las conclusiones finales al estudio realizado confirman la hipótesis orientadora del trabajo realizado.

\section{Palabras clave:}

Educación; lectura; subjetividad; literatura; cultura; escritura; investigación. between reading-education and subjectivity will be reviewed. In so doing, the main purpose is to to find differences and also add value to what the third element, the notion of subjectivity, brings in and adds to the first two. This offers very interesting and innovative research possibilities for both formal and voluntary, non-conditioned fields of education. A questionnaire has been designed and implemented in order to obtain information from students with different qualifications as well as from adults. Its aim is to contrast the opinion of those individuals with different experiences, different qualifications and age. The discussion of the results and the conclusions of the research confirm the hypothesis that has driven the research undertaken.

\section{Key words:}

Education; reading; subjectivity; literature; culture; writing; research.

\section{Résumé:}

Le point de départ de cette étude à caractère théorique et empirique est lié au potentiel éducatif de la lecture en général, et de la littérature en particulier. L'hypothèse que l'on essaie de confirmer est celle selon laquelle la littérature aide à éduquer la subjectivité.

Cet article a pour but de donner raison à la recherche orientée par la coordination lecture - éducation -subjectivité. II s'agit de la première étude réalisée dans le cadre espagnol.

On examinera les travaux qui ont étudié la relation entre les deux premiers concepts du triangle, la lecture et l'éducation, dans le but d'estimer et d'évaluer ce que le troisième concept du triangle, la notion de subjectivité, apporte pour ouvrir une voie nouvelle, puissante et innovatrice d'enseignement formel, volontaire et non conditionné.

Pour obtenir des informations, on a mis au point un formulaire qu'on a donné à des étudiants de diffèrentes matières ainsi qu'à des adultes. L'objectif était d'établir une comparaison entre les différentes perceptions de sujets ayant des expériences, formations et âges différents. L'analyse des résultats et les conclusions finales confirment l'hypothèse d'orientation du travail réalisé.

\section{Mots clés:}

Éducation; lecture; subjectivité; littérature; culture; écriture; recherche.

Fecha De Recepción: 13-3-2013
Fecha De Aceptación: 23-4-2013 


\section{Planteamiento del problema: Lectura-educación-subjetividad}

La pregunta que orienta nuestra investigación es: ¿contribuye la lectura literaria, realizada voluntariamente- es decir fuera de los cánones escolares formales obligados de transmisión- a la educación de la subjetividad de aquellos que leen? Pregunta de partida que puede clarificarse con otras muchas como las siguientes: en momentos en que las humanidades en nuestra cultura, están sufriendo hoy un fuerte varapalo: ¿nos educa o nos forma la literatura?; y si nos forma ¿de qué modo lo hace cuando la lectura se materializa voluntariamente?; ¿contribuye a construir nuestra subjetividad?... La pregunta se ubica, pues, en el terreno del aprendizaje (el del lector voluntario) y no en el de la enseñanza y su planificación (el territorio del profesor). Aún puede reforzarse la hipótesis de partida con algunas preguntas más: tal educación ¿̇tiene que ver con el potencial formativo que la literatura (la novela, la poesía, las cartas, las memorias, las autobiografías...) encierra, tal y como afirman voces autorizadas en este territorio cultural?; o ¿también está relacionado con los roles del profesor que en su día ejerció la docencia en sus clases (des)motivando a los entonces alumnos condicionados por la formalización de las enseñanzas...?; ¿acaso juegan un importante papel los métodos que tales profesores utilizan y utilizaron para enseñar la literatura, con logros frustrantes y muy contrarios a los que ellos esperaban alcanzar en sus aulas, justificando por tanto las tesis de quienes defienden que la formación literaria de las personas es tanto más fecunda cuánto más libremente se realiza la lectura de las obras literarias? La lectura a la que nos referimos en esta investigación es la que hace referencia a la literatura en el sentido amplio (novela, poesía, memorias, autobiografías...) identificando en ella lectura con literatura. El texto presente aborda cuestiones relacionadas con estas interrogaciones.

\section{Educación, literatura y subjetividad: fundamentación y antecedentes}

\section{1. ¿Tiene interés explorar las relaciones entre la educación, la literatura y la subjetividad?}

El estado actual de esta cuestión nos remite más allá de nuestras fronteras. El trabajo de la filosofa de la educación, Maxine Greene, es muy merito- 
rio en lo que respecta a la unión entre Educación-Literatura-Subjetividad en el campo de los Estudios Culturales. La autora se pregunta: ipuede la literatura unirnos al mundo real?; ¿comprender mejor el entorno que nos rodea?, ¿puede servir como instrumento incuestionable para propiciar nuestro pensamiento cuando se descubre en una obra literaria realidades alternativas a las de la "vida real" y nos percatamos de que potenciamos nuestra imaginación para construirnos otros caminos? Un texto extraído de sus conclusiones puede evidenciar la potencialidad de sus propuestas.

"Algunos jóvenes, más afortunados, tuvieron otra experiencia literaria a través de las lecturas en lugares distintos al colegio, y estas lecturas fueron para ellos las más apasionantes y las que más recuerdan. Fueron con esas lecturas y no con las que hicieron en las escuelas, con las que pudieron elaborar en su mente las imágenes de lo que ocurría a lo largo y ancho de esta o aquella geografía y cultura. Era, a través de esas lecturas, como se llegaba a conocer lo que era la amistad, el amor, la aventura, el odio, la pasión... y cuando realmente se descubrían perspectivas nuevas, mundos distintos habitados por el ser humano... En realidad "nos hacían ser distintos" de los que leían sólo las obras obligadas y preceptivas, terminábamos sabiendo cosas que nunca hubiésemos aprendido en la enseñanza reglada. (1990:252-253).

La experiencia literaria de la que habla Greene tiene lugar en condiciones particulares: se lleva a cabo voluntariamente, fuera del sistema educativo formal y reglado, alejado de pruebas y exámenes para responder a las requisitorias credendialistas, en primer lugar y, en segundo lugar, impulsan el potencial liberador y humano que encierra la literatura. El trabajo presente adopta este presupuesto como fundamentación de su tarea investigadora: nos interesa el potencial que encierra la (auto) formación llevada a cabo libremente, fuera de la planificación didáctica escolar, por motu propio y teniendo a la (lectura) literatura como recurso fundamental.

\subsection{Algunos estudios sobre la relación entre la lectura y la educación}

\subsubsection{ESTUDIO SOBRE EL BUEN O MAL LECTOR}

En "El valor de la lectura" Larrañaga, Yubero y Cerrillo, (2008), partiendo del aserto de que "ser lector requiere tener insertado el acto lector en el propio estilo de vida, en hacer de la lectura un hábito" (pág. 17), buscan 
"identificar el contenido de las representaciones sociales sobre el lector" (pág. 24). De este modo, los impulsores de este trabajo tratan de llevar a cabo el estudio en 5 universidades españolas y en 8 titulaciones. Desde una posición metodológica, distinguen entre estudiantes que hayan leído o no; es decir la situación de estudio planteada por los autores del trabajo es la de diferenciar entre sujetos lectores y no lectores para averiguar las representaciones sociales vinculadas a la lectura. ¿El método propuesto para hacerlo? La técnica de asociación libre, consistente en una evocación espontánea de rasgos en el que, a partir de una palabra inductora, se solicita a los sujetos producir expresiones o adjetivos que les vengan a la mente. Los resultados permiten la representación del buen y el mal lector, configurada con los atributos que se leen en la Tabla 1:

\section{Tabla 1}

\begin{tabular}{ll}
\hline BUEN LECTOR & MAL LECTOR. \\
\hline - Interés por conocer. & $\bullet$ No le interesa la lectura. \\
- Le gusta la lectura. & $\bullet$ Mala expresión oral. \\
- Lee mucho. & $\bullet$ No lee. \\
- Buen lenguaje. & $\bullet$ No tiene cultura. \\
- Tranquidad de comprensión. & $\bullet$ No se concentra. \\
- Inteligente. & \\
\hline
\end{tabular}

(Larrañaga, Yubero y Cerrillo, 2008; pág. 30).

Las diferencias, pues, entre un sujeto lector y otro que no lo es, se hallan en una serie de características personales que intervienen en su decisión de leer.

\subsubsection{El ESTUDIO SOBRE VALORES Y HÁBITOS LECTORES}

De carácter similar es el trabajo de I. Martínez y E. Larrañaga (2004) sobre los valores y los hábitos lectores entendiendo los valores, dentro de la psicología, como metas amplias y estables que guían la conducta de los individuos. Atendiendo a la teoría de Schwartz sobre los valores, las dos investigadoras se sirven de la escala de diez valores básicos individuales que todos los individuos (a juicio del teórico) utilizan para regular su funcionamiento social: Auto-dirección, Estimulación, Hedonismo, Logro, Poder, Seguridad, Conformidad, Tradición, Benevolencia, Universalismo. Este cuestionario está compuesto por 5 ó 6 ítems que 
inicialmente conforman 10 dimensiones de valor. La investigación se adentra en la relación existente entre los valores que mantienen los estudiantes universitarios con los hábitos lectores y las opiniones que mantienen hacia la lectura.

Para llevar a cabo esta exploración se utilizó una muestra compuesta por 111 estudiantes del Campus de Cuenca, perteneciente a la Universidad de Castilla La Mancha. A todos ellos se les administró el cuestionario de valores de Schawrtz, seguido de algunas preguntas sobre sus hábitos y opiniones sobre la lectura. El análisis de los comportamientos relacionados con la lectura se realiza a través de variables como las siguientes: opiniones de los universitarios sobre la lectura, valores relacionados con los estudios universitarios, diferencias en valores en función de algunos hábitos y comportamientos relacionados con la lectura y ,finalmente, diferencias en valores en función de las opiniones sobre lectura.

En esta misma dirección, con los valores como temática central, se presenta el trabajo de S. Sánchez y S. Yubero (2004) centrándose en los niños de educación primaria. Aquí, es el cuento el que hace de hilo conductor contribuyendo, en el caso del niño que lee, a promover su socialización: la relación entre lectura y socialización es, en este caso, "un hecho natural, complementario, al mismo tiempo irrevocable" (pág. 122), según los autores.

Aclaración: ninguna de estas investigaciones aborda el tema de la subjetividad ni la relación de ésta con la educación y la literatura. Ello legitima, aún más si cabe, el intento de profundizar en la dirección que apunta esta exploración.

\section{Herramientas conceptuales: la noción de lectura y subjetividad}

El carácter relacional de todo proyecto investigador invita a estudiar la serie de conceptos nucleares de nuestra investigación, por lo que nos aproximamos en este apartado a la idea de lectura y subjetividad.

\subsection{La idea de lectura en nuestra cultura}

Un punto de partida: Es necesario estudiar las prácticas contingentes de la lectura, sus condiciones de posibilidad materiales y semióticas -espa- 
cios, soportes, acceso, técnicas, instrumentos, competencias, ritos, gestos, etc-, entendiendo que podemos encontrar allí claves para comprender ciertas formas de subjetividad contemporánea que hacen colectivo en los que los individuos se agencian, se enganchan y se desenganchan en función de intereses, deseos y posibilidades.

Un texto no existe sino porque existe un lector que le confiere un significado; en otras palabras, la verdad de la lectura no está inscrita en el texto porque "el texto no cobra significado más que a través de sus lectores; con ellos cambia" (Birkerts, 1999).

No hay texto sin lector, pues, pero con ello no estamos privilegiando la concepción hermenéutica de la lectura como ejercicio de un sujeto soberano sino, más bien, la idea de una práctica de influencias mutuas. Si el lector se apropia del texto desde cierta capacidad y libertad interpretativa, los modos del texto generan distintas maneras de leer (distinto tipos de subjetividad lectora). La lectura es siempre una práctica encarnada en ciertos gestos, espacios y hábitos" (Cavallo y Chartier, 1998:12).

La historia de la lectura, también algunas de sus genealogías, nos muestra que la misma ha sufrido transformaciones, revoluciones provocadas por los cambios en sus soportes -del rollo al papiro, del papiro a la imprenta y de la imprenta a la pantalla-, que la han hecho pasar de una lectura con la voz (donde preeminentemente se leía en voz alta) a una lectura con los ojos (el paso a la lectura en silencio)... En definitiva, hemos asistido a distintas revoluciones o transformaciones de la lectura que nos han traído hasta nuestra actual forma de leer y de vivir en la pantalla (Albanel, 2002).

En esta última revolución de los modos de la lectura, la que configura en cierta medida las formas de nuestra subjetividad y lo que somos en este momento histórico, una nueva hipótesis exige ser problematizada. Cabría preguntarse si las nuevas formas multimodales de leer (texto, imagen fija o en movimiento, animación y audio, etc.) propias de la pantalla interactiva, no se estarán reconvirtiendo en una extensión del hablar. El papel que la imagen y la comunicación hablada han cobrado en nuestros días (televisión y cine, teléfonos móviles, chats, videoconferencias, etc.) modifican profunda y aceleradamente los soportes, los formatos y los modos de comunicación y de lectura, lo que supone la preeminencia de lo audiovisual en movimiento (teléfono móvil, imagen animada, chat, navegación) sobre la relativa quietud de lo textual (el libro impreso). 


\subsection{La noción de subjetividad: una construcción cultural y social}

Nuestra propuesta formula una concepción de la subjetividad que se ubica más allá del estructuralismo y la hermenéutica. A estas alturas se ha hecho evidente que ninguna de las dos opciones es capaz de dar cuenta del proceso de producción de subjetividad. Ahora sabemos que toda subjetividad se construye cultural y socialmente -lo que significa que es en buena medida un producto social- y también sabemos que no hay sujeto social completo. En otras palabras, la subjetividad no es exclusivamente introyección o reproducción de las representaciones culturales de una época.

$\mathrm{Ni}$ tampoco queremos reducir la subjetividad a un resultado, un producto elaborado como mera consecuencia de la expresión en el individuo humano de la ideología o las estructuras económico-sociales; pero tampoco podemos ni queremos afirmarla como reducto de autenticidad singular que expresaría una entidad autónoma diferenciada y/o enfrentada al mundo, al poder, al texto.

Así, antes que volver sobre las trilladas y complejas cuestiones sobre el sujeto y la subjetividad, y a sus ya previsibles respuestas, preferimos problematizar otros interrogantes que permitan producir otros recorridos y sentidos. Del mismo modo que la perspectiva foucaultiana no se pregunta ¿qué es el sujeto? sino ¿cómo se constituye?, la propuesta que nos interesa trata de dar cuenta de ciertos procesos heterogéneos que nos configuran a partir de distintas prácticas históricas (en nuestro caso las prácticas de la lectura) inscritas en redes de saber, de cultura y de poder en diferentes ámbitos. Así, esta es la concepción de subjetividad que se defiende en estas páginas: "Forma singularizada de ver, hacer y estar en el mundo" (Duschatky y Corea, 2005). Una definición que reclama varias matizaciones:

1. No es una definición de carácter universalista ni metafísica. Antiesencialista, la subjetividad es concebida como algo singularizante, lo que significa que existen tantas subjetividades como personas. La noción sustituye, en buena medida, al más metafísico y sustancializador de personalidad.

2. No es, por tanto, sustancia sino proceso. De ahí que no quepa una definición precisa y que en la bibliografía especializada prefiera hacerse referencia a "procesos de subjetivación" (Barabtarlo, 2009). 
3. La subjetividad se hace, en continuo tránsito, se construye y se transforma en el tiempo. Concepto que se asocia con frecuencia al de diferencia: los procesos de subjetivación propician procesos de diferenciación de y en las personas (Ema, 2009). Pero otras dimensiones como desarrollo del sentido estético, apertura al mundo, asunción de otras culturas, creencias y valores diversos, surgimiento y fortalecimiento de sentimientos y emociones..., son el resultado y la fuente de producción de subjetividad, tal y como se recogen en varios ítems del cuestionario construido para obtener información.

4. La subjetividad, por las características apuntadas, no se puede medir. Si acaso narrar o describir por cada cuál, teniendo presente que no siempre se posee el lenguaje adecuado para hacerlo y que el lenguaje, cargado de sentimientos, intuiciones, percepciones... es insuficiente para describir el mundo "interno" de las personas. Es ese sentimiento de que uno se fecunda y "crece" (por ejemplo con la lectura de obras que nos hacen gozar), que enriquece y desarrolla el horizonte vital porque la manera de pensar y de mirar el mundo es cada vez más amplia y potente (en el sentido deleuziano). Lo que nos interesaba detectar en el cuestionario que se les aplicó a la población es, justamente, ese sentimiento subjetivante, diferenciador y amplificante de las potencias de un individuo (tal y como se constata por los ítems 10,14, y 29 y sus respuestas) que se produce a partir de la lectura de obras de la literatura señaladas por él como relevantes y significativas.

\section{Diseño y metodología: la población, el cuestionario y su aplicación}

El diseño de la recogida de datos fue concebido como un muestreo por conglomerados. (Colegios, Institutos, Facultades) Los cuáles fueron aleatoriamente elegidos entre los diversos niveles educativos a los que teníamos acceso (Secundaria, Diplomaturas universitarias y Licenciaturas) y constituidos a su vez por diversos estratos de edad. La construcción del instrumento para obtener la información que buscamos sobre la relación literatura-educación-subjetividad, la hemos llevado a cabo en las siguientes fases:

En primer lugar, el grupo de discusión que promovió el diseño de la 
investigación tuvo la oportunidad de formular preguntas relacionadas con la lectura y la formación. De tal discusión se decidió identificar la percepción que voces autorizadas, expertos en literatura y educación, tienen en cuestiones relacionadas con este binomio. Ello supuso abordar la lectura de textos de especialistas reconocidos en estas cuestiones En segundo lugar, se recogieron varias ideas -algunas de ellas retraducidas en preguntas- de las cartas, diarios y memorias de escritores y literatos opinando sobre la potencialidad educativa de la literatura. En tercer lugar, de la revisión de la escasa literatura relacionada con la noción de subjetividad, se identificaron aquellas definiciones y conceptos que van vinculados a la citada noción. También esta actividad ha sido fuente de interrogaciones y preguntas.

El cuestionario debería interpelar, por tanto, las dimensiones que a nuestro juicio, componen nuestro concepto de subjetividad. Por un lado, la dimensión cognitiva, compuesta por un nivel de formación y su edad, cantidad de lecturas. Por otro lado la dimensión subjetiva constituida a su vez por componentes emocionales, estéticos y sentimientos. De estas dos dimensiones en conjunto hemos tratado de comprobar la percepción que el lector tiene de éstas en su vida y en su comportamiento subjetivo. En definitiva, en qué grado influyen en la construcción de la subjetividad y visión del mundo.

En cuarto lugar, partiendo de tales premisas se confeccionó un cuestionario, que fue aplicado a varios expertos colegas y amantes de la literatura, funcionando y usado a modo de pre-test.

De tal aplicación previa surgieron matizaciones y cambios en la redacción de algunos ítems, utilizando diversas formas de cuestiones, con escalas para su mejor entendimiento. La corrección del instrumento permitió su mejora y aplicarlo en una prueba piloto a un grupo de $3^{\circ}$ de la clase de Educación Social Especializada y a un grupo de alumnos de secundaria. De esta prueba piloto, se hizo una segunda corrección de la que surgió el instrumento definitivo, el cual se puede obtener en la siguiente dirección de internet:

https://docs.google.com/spreadsheet/viewform?hl=en_US\&formkey= dHNyUG1BRkIZazFkX01VYlhQUIBtemc6MQ\&ifq

Ya que una de las mejoras consistió en situar en internet el cuestionario de modo que obtuviéramos las respuestas de los diversos conglomerados tanto directamente, como desde sus hogares o aulas, de manera voluntaria y cuando la ocasión les permitiera. Los conglomerados se de- 
finieron como personas estudiantes de Secundaria, estudiantes universitarios y personas mayores. A todos ellas se les supone un nivel cognitivo suficiente para acceder a la lectura sin dificultad.

El total de la población encuestada: 580 personas que han respondido voluntariamente, sin ningún tipo de presión.

\section{Tabla de resultados}

Los siguientes datos han sido obtenidos gracias a las herramientas que nos ofrece https://docs.google.com.A través de una cuenta en GMAIL, se puede acceder a una serie de programas que permiten la confección de dibujos, hojas de cálculo, o un formulario que puede ser cumplimentado desde cualquier sitio de Internet, y cuyos datos se van anotando en una hoja de cálculo adjunta. De este modo, una vez completada la muestra que se pretende obtener, se pueden descargar los datos obtenidos a través de diversos formatos, para su posterior descripción o tratamiento estadístico. Esta forma de investigar constituye una forma rápida, muy económica y potente para obtener datos relevantes sobre un tema determinado.

No hemos pretendido, tal como se decía en la introducción de este trabajo, realizar un análisis exhaustivo de los datos y opiniones obtenidas, ya que se trata de un tema de interpretación y de carácter eminentemente subjetivo. No obstante gracias a estos 580 formularios, es posible dar una amplia panorámica de la influencia de la literatura en la formación de la subjetividad de las personas.

El enlace por el cual se accede al formulario es el siguiente:

https://docs.google.com/spreadsheet/viewform?hl=en_US\&formkey= dHNyUG1BRkIZazFkX01VYlhQUIBtemc6MQ\&ifq

A continuación de estos datos ofrecemos los resultados obtenidos en el tratamiento de lo respondido en los cuestionarios.

\section{Comentando los resultados}

Una vez localizado en Internet con el enlace citado más arriba, podemos observar y seguir fácilmente los resultados obtenidos y entender la relación entre los diversos ítems y las dimensiones que a continuación se explican. 
El cuestionario, de las dimensiones aludidas más arriba (la dimensión cognitiva y la subjetiva) trata de comprobar la percepción que el lector tiene de éstas en su vida y en su comportamiento subjetivo.

Para ello hemos planteado una serie de cuestiones cuya valoración se realiza utilizando escalas de 1 a 10 (Preferencias de género), Escalas de respuesta múltiple (Expresión de sentimientos ) escalas binarias de "mayor - menor" ( Emociones sentidas) escalas de valoración 1-3 (Grado de influencia en su vida) escalas múltiples y respuestas de tipo abierto (Sentido estético e influencia de la lectura en su percepción) y finalmente una escala binaria [si-no] en la percepción del grado de relación entre Educación y Desarrollo de la subjetividad.

Los resultados no están en modo alguno completos, podríamos haber incluido en el cuestionario algunas cuestiones para delimitar resultados por disciplinas, sobre la disponibilidad de tiempo de los distintas edades, profesión, ... pero no es el objetivo de esta investigación preliminar hacer inferencias estadísticas, sino describir y valorar, cualitativamente, la relación percibida por los lectores entre sus lecturas y el desarrollo de la propia subjetividad, como una confirmación o negación de las hipótesis de partida.

Los resultados son comentados según el orden de las preguntas en el cuestionario.

1. ${ }^{a}$ Cuestión Formación Académica.

Rango de edad $1=$ (Hasta 21 años)

Total 316

Rango de edad 2 $=($ Desde 21 a 40 años $)$

Total 194

Rango de edad 3= (Mayores de 40 años)

Total 71

Como se ve el mayor número de entrevistados (316) han sido personas muy jóvenes (desde los 16 a los 21 años) con un nivel de estudios de Bachillerato o equivalente. Otra gran parte de la muestra (194) lo componen personas que tienen entre 21 y 40 años, integrada por estudiantes universitarios con un nivel de 'Diplomado'.

El resto (71) está integrado por personas adultas y mayores que tienen en su mayoría una gran cantidad de libros leídos (>de 50) y tienen titulaciones de 'Licenciatura' o están en vías de serlo.

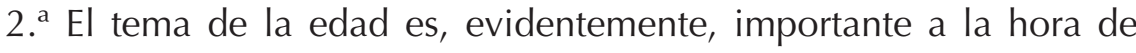
saber desde cuándo se ha empezado a leer y cuándo se ha dejado de hacerlo, o simplemente cuánta lectura se vincula a la edad si es que realmente se puede llevar a cabo esta asociación.

De los tres rangos de edad que se utilizan el más numeroso es el de los jóvenes (el $n^{\circ} .1$ ) y de los universitarios (rango $n^{\circ}$. 2). Sin embar- 
go, las personas mayores que han respondido (rango $\mathrm{n}^{\circ} 3$ ), 71 personas, muestran haber leído bastante: faltaría saber, por contraste con los otros rangos de edad, si ello responde a la "disponibilidad de tiempo" para la lectura, a la prioridad que se le da a este acto o a otras razones como, por ejemplo, el nivel de estudios alcanzado.

3. ${ }^{a}$ En lo que respecta al nivel de estudios. Los grados de formación escolar son altos, impensables hace tan solo medio siglo. Lo que no quiere decir que los que se han quedado en el graduado escolar o en el certificado escolar no lean o lean menos sino que, en una interpretación directa, los que han respondido mantienen esta relativa alta acreditación formal: el $65 \%$ del total, 375 personas, poseen o estudian un nivel de bachillerato y el $17 \%$ y $16 \%$ respectivamente (99 y 92 personas) son diplomados y licenciados.

4. ${ }^{a}$ Era previsible que los autores más leídos en la cultura contemporánea española fueran los internacionales y, desde luego, los más actuales o, por matizar aún más los autores del siglo XX, salvo algunas excepciones que gustan de la tradición literaria. Es decir, el 70\% de los encuestados preferían autores internacionales como Ken Follet, Stieg Larsson, Jostein Garder... y el 29\% nacionales como Lorca, Miguel Hernández, María Dueñas...

$5 .^{\circ}$ De todos los respondientes 199 , es decir, el $35 \%$ del total han leído más de $50 \%$ libros; 213 , el $37 \%$ entre 20 y 50; 160, el $289 \%$ entre 0 y 20 . Ello quiere decir que hay personas que leen bastante y que la pasión por la literatura constituye una de las características que identifica la subjetividad de los respondientes. Falta por saber, ateniéndonos al criterio de edad, si leer más o menos tiene que ver con el tiempo vivido.

$6 .^{\circ}$ El género que más les gusta a las personas que respondieron al cuestionario es el de aventuras, seguido del romántico, el histórico, la ciencia ficción: estos géneros son los más votados por los lectores respondientes. La diferencia con otros géneros citados (policiaco, novela negra, biográfico y autobiográfico) no es muy grande; sí con los géneros bélicos y epistolar. En este punto, la edad imperante de la población, la juvenil, debe haber sido decisiva a la hora de expresar una valoración global de las respuestas.

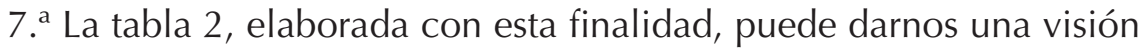
comprensiva de las respuestas dadas a una valoración por género de 1 a 10 (máximo valor a la cuestión). 
Tabla 2

\begin{tabular}{|c|c|}
\hline Género & Comentarios a las puntuaciones obtenidas \\
\hline POESIA & $\begin{array}{l}\text { Los datos resultantes muestran valores opuestos polarizados y } \\
\text { simétricos con respecto a la media (5) y énfasis tanto en nivel } \\
\text { "3" como en " } 8 \text { " no discriminando una respuesta concreta. }\end{array}$ \\
\hline *Ciencia Ficción & $\begin{array}{l}\text { Gran nivel de aceptación que acumula una puntuación total } \\
\text { de } 60 \text { puntos a partir del nivel de impacto " } 5 \text { " hasta el " } 10 \text { " }\end{array}$ \\
\hline *Romántico & $\begin{array}{l}\text { Un poco más aceptación que el anterior género acumulan- } \\
\text { do una puntuación de impacto de } 64 \text { a partir de la media (5) }\end{array}$ \\
\hline Bélico & $\begin{array}{l}\text { Muy bajo nivel de aceptación de este género con } 48 \text { puntos } \\
\text { por encima de la media y con puntuación "0" en el ítem } 10 .\end{array}$ \\
\hline *Aventuras & $\begin{array}{l}\text { Es el que a juicio de los entrevistados ofrece mayor acepta- } \\
\text { ción al obtener una puntuación de } 84 \text { puntos por encima de } \\
\text { la media. }\end{array}$ \\
\hline Policiaco & $\begin{array}{l}\text { El género policiaco no presenta un impacto destacable con } \\
\text { una puntuación de } 53 \text { por encima de la media }\end{array}$ \\
\hline Novela Negra & $\begin{array}{l}\text { Similares datos obtenemos con la Novela Negra con } 40 \\
\text { puntos y sólo } 5 \text { personas han respondido con } 10\end{array}$ \\
\hline *Biográfico & $\begin{array}{l}\text { No ocurre así con este género en el que la respuesta indica } \\
\text { un gran impacto al obtener una puntuación de } 71 \text { puntos } \\
\text { por encima de la media y } 56 \text { de ellos acumulados entre el } \\
\text { nivel } 7 \text { al } 9 \text {. Tal vez por el tema de que se trate y por poder } \\
\text { identificarse con la biografía aparece este nivel de acepta- } \\
\text { ción en este género. }\end{array}$ \\
\hline Autobiográfico & $\begin{array}{l}\text { En cambio en este género no se da el mismo impacto, acu- } \\
\text { mulándose en el valor central (5) por lo que las respuestas } \\
\text { no son discriminantes. }\end{array}$ \\
\hline Histórico & $\begin{array}{l}\text { Claramente el género histórico es el que ha causado la } \\
\text { mayor aceptación en los lectores, acumulando sólo en los } \\
3 \text { valores superiores }(8,9,10) 51 \text { puntos y si tomamos en } \\
\text { cuenta los que están por encima de la media (5) } 85 \text {, como } \\
\text { dato significativo, observemos que nadie pulsó en el nivel } 1 \\
\text { de impacto. }\end{array}$ \\
\hline Correspondencia & $\begin{array}{l}\text { Este género no conlleva un nivel notorio, al juzgar por las } \\
\text { respuestas, y hay un elevado número de lectores (11) que } \\
\text { señalan el nivel "1", indicando tan sólo } 2 \text { personas un nivel } \\
\text { "10" de agrado. }\end{array}$ \\
\hline Memorias & $\begin{array}{l}\text { Igual ocurre con el género "memorias", que destaca en un } \\
\text { elevado número } 25 \text { en el nivel de aceptación " } 5 \text { " de modo } \\
\text { que no determina una tendencia clara. }\end{array}$ \\
\hline
\end{tabular}


La lectura de obras históricas y de aventuras domina. Los otros géneros, no alcanzan tal impacto por ser demasiado especializados o por ser historias de personajes muy concretos. El romanticismo como contrapunto muestra un grado que nos esperanza ante una sociedad cada vez más pragmática y tecnocrática.

8. ${ }^{a}$ De los sentimientos provocados por la lectura son la alegría, el amor, la admiración, la ternura, el optimismo, la esperanza, la compasión, la lástima, y el deseo los más valorados. Todos ellos sentimientos positivos. A medio camino quedan sentimientos como el gozo, la lealtad, la soledad, la envidia y el miedo. Y en territorio bajo menos del $20 \%$ : culpa, egoísmo, rencor, venganza, soberbia, crueldad. La literatura es, pues, asociada a valores positivos, lo que permite pensar una cierta articulación, a su vez, entre la literatura, la educación y la subjetividad.

9. ${ }^{\circ}$ Curiosamente, como en los ítem que remiten a los sentimientos, los respondientes valoran con intensidad mayor aquellas emociones que consideran positivas, como el disfrute, el asombro, la curiosidad, el interés, la serenidad, el contento, la gratificación, el amor, la pasión,... y, por el contrario, valoran con intensidad menor aquellas emociones que podrían considerarse negativas, tales como inseguridad, temor, angustia, insatisfacción, indiferencia, frustración, decepción, pesimismo, desolación, rabia, resentimiento, la ansiedad, furia...

$10 .^{a}$ Sobre la influencia de la literatura en la formación, el 69\%, (400 personas), reconoce, afirmativamente, que tal influencia existe, es cierta y se produce con la lectura, durante y después de ella. Los que creen que tal influencia es escasa sólo son el $27 \%$, y el $1 \%$ piensa que no existe tal hecho. La diferencia entre el sí y el no es, pues, muy grande. La pregunta, pues, que hace de pórtico de esta investigación -si la lectura/literatura contribuye a la formación de la subjetividad- es respondida positivamente por el grueso de la población respondiente.

11. ${ }^{a}$ Sin embargo, a la pregunta de si crees que las lecturas influyen a la hora de tomar decisiones, el número de afirmaciones baja hasta un $42 \%$. Por contra, aumenta hasta un $50 \%$ la cantidad de los que creen que esta capacidad de decisión potenciada por la literatura es escasa, muy poca.

12. ${ }^{a}$ Las dos preguntas anteriores y las posteriores se relacionan profundamente con este ítem que se interroga sobre la lectura y su inducción a pensar, decidir, actuar, interpretar el mundo y comprenderlo así como desarrolla el espíritu relacional. Los tantos por ciento en cada una 
de estas variables son diferentes pero todos ellos mantienen una media alta. En suma, pensar, entender e interpretar el mundo y relacionarse en él van de la mano; tienen que ver todas ellas con actividades reflexivas del ser humano. La subjetividad humana va muy unida a ellas.

13. ${ }^{a}$ Las respuestas a este ítem tenía que ser diversa debido a la misma naturaleza de la pregunta. Las obras que han impactado a los respondientes son casi siempre diferentes en cada caso. Los gustos por la lectura también lo son.

14. ${ }^{a}$ No muy lejana de los ítems anteriores, la pregunta 14 insiste en explorar las diferentes dimensiones de la subjetividad que venimos explorando. En este caso, el $86 \%$ ha respondido que la lectura potencia la imaginación. El 8\% piensa que esto no es cierto. Lo que no sabemos es qué pasa con el $6 \%$ restante. Parece que la respuesta a esta cuestión debe estar en el absentismo de algunos encuestados.

15. ${ }^{a}$ La misma situación se repite con este ítem. El $72 \%$ del total de encuestados creen que la lectura le ha abierto nuevos horizontes y el $22 \%$ piensa que no. No obstante, el nivel de afirmación baja en esta pregunta; no se encuentra siempre relación causal entre el poder de la lectura y la apertura a diferentes horizontes. Al menos eso es lo que piensan 128 de las 580 personas que constituyen la población encuestada.

16. ${ }^{a}$ ¿Ha promovido la lectura tu capacidad de abordar la realidad?; a esta cuestión ha respondido afirmativamente el $69 \%$ y negativamente el $24 \%$. La posición sigue siendo afirmativa, como en el caso de los últimos ítems anteriores, ya que en conexión con ellas la lectura promueve la interpretación y la comprensión de la realidad y, por ende, la de abordarla.

17. ${ }^{a}$ Ha sido el $63 \%$ de los encuestados los que han respondido afirmativamente a la relación lectura como facilitadora o promotora de sentido de la justicia, de la honradez, de la libertad. Como en las preguntas anteriores se trata de explorar los caminos de constitución de la subjetividad. El 33\% piensa que no existe tal relación y que la lectura no fomenta el sentido de estos valores.

18. ${ }^{a}$ Respuesta similar ha tenido esta pregunta que se preocupa por saber si la literatura, en particular, y la lectura en general, promovía nuevas vivencias en el lector. Es plausible pensar que, con un recurso educativo tan potente como la literatura, la producción de vivencias, a través de la imaginación o el abordaje de la realidad por ejemplo, era un efecto claro.

19. ${ }^{a}$ Este ítem insiste, como los anteriores, en explorar la dimensión 
axiológica de la subjetividad humana. El 69\% piensa que estos valores han adquirido relieve en sus vidas y en ello ha tenido mucho que ver la lectura; un 24\%, por el contrario, no comparte esta opinión.

$20{ }^{a}$ Como en éste, formulado para saber si emociones nuevas y sentimientos diferentes son provocados por la lectura: el $73 \%$ piensa que sí y el $20 \%$ que no. Lo que es raro, en este último caso, porque los tiempos de la lectura, normalmente tiempos de enganche y vibraciones suelen ser, también, de emociones y sentimientos.

$21 .^{a}$ Las dudas que pueden haberse despertado en las preguntas inmediatamente anteriores, no desaparecen en las respuestas a este ítem. El $74 \%$ piensa que con la lectura se penetra en otras culturas, con sus valores y creencias y el $19 \%$ que no.

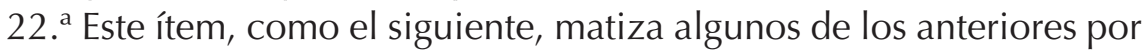
lo que son ítems "tramposos"; en realidad estaban formulados anteriormente con otro lenguaje. El $66 \%$ piensa que la lectura si ha sido promotora de libertad y de apertura y el $27 \%$ se niega a confirmar tal relación.

23. ${ }^{a}$ Una pregunta inevitable era si la lectura te abre a nuevos mundos, si te facilita el conocimiento de nuevas situaciones diversas y enriquecedoras. Aquí también no nos hemos sorprendido con las respuestas ya que el $78 \%$ ha contestado afirmativamente y el $14 \%$ que no. En realidad este ítem es una ampliación del 18. Las respuestas positivas han sido numerosas.

$24 .^{a}$ Esta pregunta, desglosada en 7 ítems trata de vincular la subjetividad al desarrollo del sentido estético. Atendiendo al desglosamiento realizado, organizamos los resultados obtenidos en función de cada una de las aportaciones de la lectura a las vidas de los respondientes. Las respuestas se valoraron de 1 (nada) a 4 (mucho) pasando por 2 (poco) y 3 (bastante). Sumados los muchos y los bastantes de cada uno de ellos, puede decirse que la lectura desarrolla la sensibilidad como la competencia para apreciar la belleza, fomentar la creatividad y estimular la imaginación, promover sentimientos y provocar emociones así como impulsar el enriquecimiento del vocabulario y el logro de una mayor fluidez expresiva.

25. ${ }^{a}$ Las respuestas a este ítem han sido contundentes. Para el 90\%, efectivamente, la lectura ha sido un motor de curiosidad, una invitación a seguir leyendo. Parece cumplirse el supuesto de que "Cuanto más lees, más quieres leer". Solo el 9\% se niega a creer que la motivación por la lectura provenga de la misma lectura. 
26. ${ }^{a}$ Este ítem pone en juego la filosofía de este cuestionario o trata de confirmar si, verdaderamente, la opción de leer voluntariamente es un supuesto con visos de realidad o no. Las respuestas dadas muestran que el $66 \%$, solo este tanto por ciento, prefiere no acceder a la literatura a través de la enseñanza formal planificada. Y, curiosamente, un relativo alto porcentaje, el 31\%, sí que prefiere el formato disciplinar.

$27 .{ }^{a} Y$ es que, a lo largo de la vida de los respondientes, el $75 \%$, tuvieron oportunidad de leer literatura conducida por profesores que les motivaban a leer. Estas respuestas no contradicen el hecho rotundo de que leer propicia el leer más. Durante el periodo de formación se ha podido tener profesores motivadores en una materia o no tenerlo; haberlos tenido en el campo de la literatura o no. En cualquier caso, el $23 \%$ de respondientes no tuvieron, durante su formación, educadores que les engancharan al placer de leer. Si lograron tal enganche se debió a otros canales de formación.

28. ${ }^{a}$ Profundiza dos ítems, el 26 y 27. Acorde al hecho de que un buen número de respondientes hayan tenido la oportunidad de tener buenos profesores que les enseñaron a leer puede pensarse que ello se debió a que tales profesionales de la formación utilizaron metodologías educativas con éxito, logrando que sus alumnos amaran la lectura. El $56 \%$ piensa así. Sin embargo, el 35\% responde que el hecho de que tuvieran profesores que les motivaron a leer no significa que utilizara en sus clases buenos métodos pedagógicos para hacerlo.

29. ${ }^{a}$ La última pregunta retoma la tesis que orientó el diseño de este proyecto de investigación así como la construcción de este cuestionario. El 92\% de los respondientes prefieren acceder a la literatura libre y voluntariamente. Incluidos aquellos que tuvieron buenos profesores que les enseñaron con adecuadas metodologías educativas. El 6\% no comparte este criterio y permite pensar que quieren seguir teniendo la oportunidad de guías para acceder a la lectura.

Finalmente, para visualizar mejor los resultados obtenidos, hemos creído conveniente la elaboración de una tabla que relacionen los ítems del cuestionario con las dimensiones que integran nuestro concepto de subjetividad: 


\section{Figura 1}

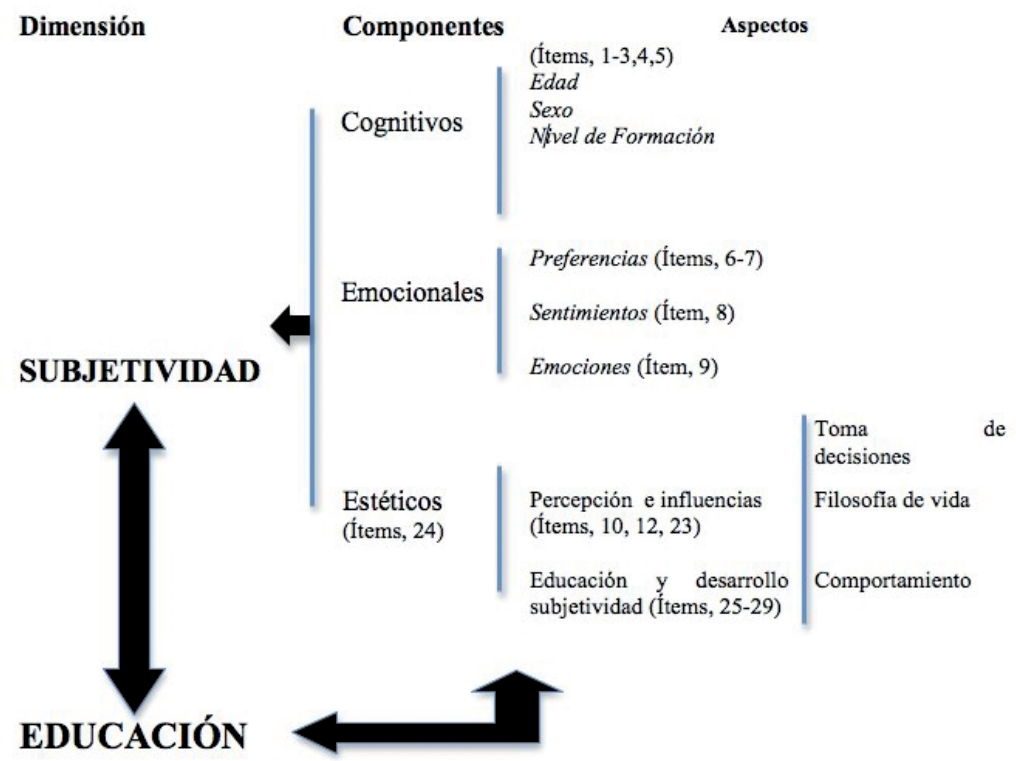

\section{Algunas conclusiones}

A la vista de la búsqueda teórica realizada, y de la exploración empírica cuyos resultados acabamos de comentar, podemos formular algunas conclusiones, siempre provisionales, de esta investigación orientada a averiguar si la lectura contribuye a la construcción de la subjetividad.

Primera. Efectivamente, la lectura en general y la literatura en particular son potentes dispositivos pedagógicos en el desarrollo de la subjetividad humana. Según los resultados obtenidos esta contribución es tanto mayor cuanto más esta lectura se haga voluntariamente. En este sentido, las respuestas fueron contundentes.

Segunda. El hecho de que la voluntariedad, por decisión personal, sea una variable fundamental no oculta ni soslaya que la literatura desde un enfoque disciplinar también es motor de subjetividad, tal y como algunas respuestas apuntan. Esto supone reafirmar el valor de la literatura en su dimensión formalizada y planificada por los profesores en las aulas. Este hecho debería ser considerado por la política educativa y el sistema educativo que enfatiza la necesidad de la educación ciudadana: la literatura en tanto que creadora de subjetividad puede ser un factor 
relevante de formación cultural de una ciudadanía enriquecida en valores, emociones y sentimientos, en la toma de decisiones y asunción de responsabilidades.

Tercera. La literatura preferida es la contemporánea más que la clásica, aunque ésta no sea absolutamente negada. Esta conclusión debería tenerse presente; sobre todo si se piensa que, en los centros donde se imparte como asignatura, la literatura suele ser enseñada desde un punto de vista histórico deteniéndose en aspectos que parece no interesar excesivamente a los estudiantes, como escuelas, autores, fechas...

Cuarta. El potencial educativo de la literatura se encuentra en la lectura en sí, en el modo en que se lee y se accede a todos los valores que ella encierra para el ciudadano normal, aquél que no pretende el dominio de un campo de conocimiento sino disfrutar de los productos de la cultura.

Quinta. Que la literatura contribuye a educar la subjetividad es una hipótesis que se confirma ya que, declaradamente, promueve sentimientos y emociones, incluso, impulsa valores estéticos que enriquecen los modos de pensar y de actuar las personas a lo largo de sus particulares procesos de construcción personal y de diferenciación identitaria a lo largo de sus vidas.

Sexta. Estas tesis se ve aún más reforzada por otro hecho significativo: en las respuestas a la lista de emociones y sentimientos que se defienden como promotores del desarrollo de la subjetividad son claramente elegidas aquellas que presentan un carácter más positivo.

Séptima. Además de propiciar emociones y sentimientos también la literatura, en su contribución a enriquecer los procesos de subjetivación de las personas, impulsa capacidades como tomar decisiones, pensar, actuar, entender el mundo, interpretar, abrirse a nuevas formas de relación...

Octava. La mayoría de los encuestados reconocen que alguna obra literaria, en algún momento de sus vidas, les produjo un verdadero impacto, en casi todos los casos produjeron una transformación en su devenir subjetivo. No siempre se sabe explicar en qué consiste esta transformación aunque se reconoce que se ha producido. Algo "ha cambiado" en ellos.

Novena. No hay duda alguna que la literatura potencia la imaginación y fomenta la creatividad así como diversos valores estéticos. Esta tesis por sí sola debería bastar para destacar la importancia de la literatura en cualquier cultura asertiva que se precie al tiempo que convoca a los 
gestores de la educación a no desaprovechar este potencial pedagógico que propicia la imaginación.

\section{Limitaciones del estudio y prospectiva}

El estudio realizado ha estado vinculado, como se ha apuntado anteriormente, a dos ambiciones declaradas y consecuentes: $1^{\text {a }}$ confirmar, tal y como han mostrado los resultados, que la lectura es potenciadora de subjetividad, y $2^{\mathrm{a}}$ proponer una investigación histórico-genealógica de los modos de lectura desde la modernidad hasta nuestros días atendiendo a los dispositivos de subjetivación que configuran.

La primera ambición, permite entender que el estudio empírico realizado no haya querido explotar todos los datos obtenidos ni tampoco propiciar otros nuevos (por lo que quedan variables que no han sido atendidas: comparar resultados en función del tipo de estudios que realizan los estudiantes, también introducir la variable profesión con la finalidad de comprobar si van vinculadas a un tipo de subjetivación o no, codificar con más precisión las diferencias y aproximaciones de los sentimientos y emociones....); la segunda ambición, apuesta por otro tipo de investigación en la que se puedan cartografiar algunos de los dispositivos de lectura privilegiados por los estudiantes de diferentes niveles así como analizar las tecnologías y prácticas de subjetivación a través de la lectura tal y como se manifiesta en tres dispositivos contemporáneos en los que hoy se lee: el dispositivo aula, el dispositivo pantalla y el dispositivo libro. Ello nos permitiría realizar un balance de las problematizaciones que los análisis cartográficos realizados plantean en lo relativo a las nuevas formas de configuración de la subjetividad.

\section{Referencias bibliográficas}

ALBANELL, P. y otros (2002). Hablemos de leer. Madrid: Anaya.

BARABTARLO, A. (2009) (coord.). La historia de vida. El encuentro con nuestra subjetividad. México: Castellanos editores

BIRKERTS, S. (1999). Elegía a Gutenberg. El futuro de la lectura en la era electrónica. Madrid: Alianza.

CAVALLO, G. Y CHARTIER, R. (1998). Historia de la lectura en el mundo occidental. Madrid: Taurus. 
El potencial educativo de la lectura en la construcción de la subjetividad

juan Sáez Carreras, Margarita Campillo Díaz y José García Molina

DUSCHATZKY, S. y COREA, C. (2005): Chicos en banda, los caminos de la subjetividad en el declive de las instituciones. Buenos Aires: Paidós.

EMA, J.E. (2009): "Capitalismo y subjetividad. ¿Qué sujeto, qué vinculo y qué libertad?". En Psicoperspectivas, vol.8 (2); pp.224-247.

FOUCAMBERT, J. (1989). Cómo ser lector: leer es comprender. Barcelona: Laia.

GREENE, M. (1990). Realizing Literature’s Emancipatory Potencial. En J. MEZIROW. Fostering Critical Reflection in Adulthood. San Francisco: Jossey Bass.

LARRAÑAGA, E.; YUBERO, S. Y CERRILLO, P. (2008). Estudio sobre los hábitos de lectura de los universitarios españoles. Madrid: CEPLI.

LARROSA, J. (2003). La experiencia de la lectura. Estudios sobre literatura y formación. México: FCE.

MARTÍNEZ, I. Y LARRAÑAGA, E. (2004). Un estudio sobre valores y hábitos lectores en jóvenes universitarios. En S. YUBERO, E. LARRAÑAGA Y P. C. CERRILLO (Coords.) Valores y lectura: Estudios multidisciplinares Cuenca: UCLM; pp.65-87.

SÁEZ, J. (1999). La capacidad transformadora de la literatura: educación y conocimiento. En SÁEZ, J. Y CAMPILLO, M. (coords.). Cuentos pedagógicos. Relatos educativos. Murcia: D.M; pp.9-31.

SÁNCHEZ, S. Y YUBERO, S. (2004). La transmisión de valores desde la lectura. Un estudio con niños de educación primaria. En S. YUBERO, E. LARRAÑAGA Y P.C. CERRILLO. (coords.) Valores y lectura. Estudios multidisciplinares. Cuenca: UCLM; pp. 89-129.

YUBERO, S, LARRAÑAGA, E. Y CERRILLO, P. (2004). Valores y lectura. Estudios multidisciplinares. Ediciones de la Universidad de Castilla La Mancha. 